\title{
Literature Review of Dual Data Hiding and Image Compression and Digital Inpainting
}

\author{
Sarita S. Kamble ${ }^{1}$, A. S. Deshpande ${ }^{2}$ \\ ${ }^{1,2}$ E \& TC, ICOER Pune, India
}

\begin{abstract}
This paper presents a review of dual data hiding and image compression techniques and digital inpainting. Reversible data hiding plays important role in information security. A technique image compression is followed by data hiding scheme. Data hiding and image compression is two independent modules at user and receiver side. A method of digital inpainting is used to remove/replacement of unwanted objects of media. Digital inpainting consists of inpainting of audio, video and image. The goal of any digital image inpainting algorithm represents a reconstruction of the misplaced or scratched regions in a visually plausible way. First the Classification of reversible data hiding techniques are introduced, then types of digital inpainting methods. There are various applications of data hiding techniques and digital inpainting. Also advantages and limitations of these methods are discussed in detail.
\end{abstract}

Keywords: Reversible data hiding, image Compression, digital inpainting

\section{Introduction}

The magnificent improvement of communication network technologies, it becomes very easy to communicate with each other via the internet. In communication the important factors are usage of networking bandwidth and information security. Information security and transmission bandwidth is vital topic of research. The various techniques of secret data hiding can be achieved by compression. Steganography, watermarking, cryptography are the ways of information security. Most of the technique limited to visual quality of media after extraction. Recently, many data hiding and image compression techniques are available. These are used to reduce storage space and also for transmission bandwidth. The combinations of various data hiding and image compression techniques give result which is measured in terms of compression ratio, peak signal noise ratio etc.

Firstly data hiding techniques included invisible ink, secret writing using chemicals, templates laid over text messages, microdots, changing letter/word/line/paragraph spacing, changing fonts. Data hiding technique is followed by image compression algorithms. Both data hiding and image compression are the two independent modules. The process of data hiding has two important aspects. One is group of embedded data and another is cover media. Media would be image, audio or video. Least Significant Bit (LSB) [10] is one of the popular data hiding technique which based on manipulating LSB. It can be achieved by directly secret bits are replaced by LSBs of cover image.

Inpainting is an ancient art of modifying an undetectable form. Diffusion [14] is the first digital inpainting techniques. According to the technique lost region is packed by diffusing the image information. The acknowledged region is filled into the missed region which is based on manipulating of the pixel. Image inpainting is a part of digital inpainting. Now days, various movies and videos possess digital special effects such that removal of unwanted objects, insertion of virtual objects red eye removal and composition of graphics etc. All those operations are performed by using special graphic software. Main aim of inpainting is to restore a damaged paintings and images/videos to the removal or alternate of chosen objects. Various methods are available such as diffusion based inpainting, texture synthesis, PDE inpainting, Exemplar inpainting etc. Inpainting has various applications like retrieval of super imposed text like names, subtitles, symbols or advertising; restoration of ancient documents, photographs, red-eye removal, multimedia editing and damaged films.

The remaining paper is structured as follows. In section-2, combined data hiding and compression techniques are described. Reversible data hiding guarantees that original image can be rebuilt completely after extraction and viceversa. The digital inpainting techniques classification is discussed with their merits and demerits in section-3. The data hiding schemes in terms of performance criteria and various applications are discussed in section-4. Conclusion and future scope of various data hiding techniques and digital inpainting are mentioned in section-5.

\section{Dual Data Hiding and Image Compression}

Du and Hsu, [18] proposed an adaptive data hiding method for VQ compressed images in 2003. The process of embedding data depends upon secret bits. Here, the vector quantized codebook was segmented into two or more sub codebooks, and the most excellent match in one of the sub codebooks used to hide secret data. Drawback of this method is that it has low embedding capacity.

Chang and Wu in 2006 [19] proposed a codeword clustering technique [19] can be used to improve embedding capacity. The adaptive clustering process uses codeword order succession method to insert secret data into the VQ index table. This allows proper codeword substitution so that embedding capacity gets increased.

Lin et al. in 2009 [20] proposed adjustment of the known distance threshold has been done according to the required hiding ability. A similar number of codewords are grouped to embed the secret sub-message. The proposed algorithm is separated into three segments, a bunch of codeword segment consists of two most similar codewords are grouped together 


\section{International Journal of Science and Research (IJSR) \\ ISSN (Online): 2319-7064 \\ Index Copernicus Value (2013): 6.14 | Impact Factor (2014): 5.611}

which don't belongs to any group. Similar codewords are added to it. Another segment, each codeword is assigned to embed a certain sub message and length is nothing but number of codeword in the group, called embedding segment. Therefore, embedding capacity increases with number of codeword in the group. In the extracting segment, order of codeword is useful to determine embedded message. The experimental outcome shows that good embedding capacity than previous methods.

Chaur Heh Hsieh and Jyi Chang Tsai proposed a search order coding [21] algorithm. The technique is capable for compress of the VQ index table. A new lossless algorithm that exploits the inter block relationship in the index field. A method compares existing index with earlier indices in a predefined search path and send it to the decoder. It is capable to achieve better result for bit rate through searching close by similar picture block. It is simple and efficient method to reduce bit rate.

\subsection{Reversible data hiding techniques}

Barton's in 1997 [4] proposed a reversible data hiding technique; scheme compresses the bits to be biased by the embedding process then hides the compressed data. Arbitrary digital information is embedded within a stream of digital data in such a way that only user knows modified digital information. During recovery, original bits are decomposed and are used to restore customized bits for reconstruction of unique block.

Fridrich, et al. [5] proposed an reversible water marking scheme used for authentication of digital images in JPEG domain. Small amount of distortion is observed in previous authentication watermarking schemes. Firstly, an invertible watermarking scheme method removes distortion due to authentication to get original image. The method possesses lossless compression of influenced bit stream resulting from quantized JPEG coefficient. Second method the invertible water marking based on modifying quantization table to prevent loss of a bit per DCT coefficient during the embedding procedure. Both the techniques are fast and used for medical as well as military applications.

In 2003, Xuan, et al. [16] explored a large capacity distortion free data hiding system related to integer wavelet domain. The structure can be applied to wavelet based JPEG2000 compressed image.

Yang, et al. 2005 [17] developed a reversible water marking method based on modified vector quantization (VQ). While encoding, adjacent blocks are used to encode present blocks, but extra flag bits are required. Reversibility is the advantage of this method where as method degrades visual quality and compression ratio.

\subsection{Least significant bit}

The most popular spatial data hiding method is least significant bits substitution (LSB). In 2001, Fridrich et al. [5] proposed a least significant bit (LSB). The least significant bits of a byte are replaced with number bits of the secret message. It is not visible to the human eyes. In this method end bits of message string are replaced by secret bits. Remaining bits are as it. There is slight variation in color which is indistinguishable to human eyes.

\section{Digital Inpainting}

Digital inpainting is main branch of image inpainting. It consists of fully repeated detection and removal of scratches in motion picture [22-23]. Digital automatic inpainting uses a software tool allows sophisticated way to user but mostly manual processing tool [24].

There are several approaches for inpainting which can be classified as follows: PDE based inpainting, Texture Synthesis based Inpainting, Exemplar Inpainting, partially automatic etc.

\subsection{PDE based Inpainting}

PDE [14] based inpainting works on lines at border of the region. It is iterative process of inpainting gives best result on border region than inner region. This method is applied for missed regions and small target area with no texture. Drawback of this method is, it is time taking process for large area and blurring effect is observed.

\subsection{Texture Synthesis based Inpainting}

The Texture based synthesis [14] is a field related to inpainting. The idea of texture synthesis is that an input sample of a texture is given, and the aim is to produce more of that texture. The simplest solution is to tile the texture sample on a rectangular grid of desired size. However, even if the sample can be tiled seamlessly, the resulting larger grid structure is easily visible and it distorts the perception of the actual texture. A deterministic or structured texture is characterized by a primitive element that is regularly placed on a grid or a lattice. For example, floor tiles, brick walls are regular textures. The texture synthesis based on approximation of texture. These algorithms are not suitable for natural images cause of their composed structures. Hence the merit of the texture synthesis techniques in Inpainting, it uses only a small division of inpainting and this method is not suitable for a large object.

\subsection{Exemplar Inpainting}

Exemplar inpainting [14] is very subjective and effective approach to reconstruct large target region. It is explained in two steps; first step is setting a priority which portion will be inpainted first. And next step consists of selection of similar patch. This is effective inpainting approach because it samples the corresponding patches from known region. Matching of patches will be done by certain matrices like length, width, color etc.

\subsection{Partially Automatic inpainting}

Jian et.al [15] termed as inpainting with Structure propagation. This can be performed by two step process. Firstly, manually find out significant lost information by sketching object boundaries from the known to the unknown region. Texture is generated by using patch based texture 


\section{International Journal of Science and Research (IJSR) \\ ISSN (Online): 2319-7064 \\ Index Copernicus Value (2013): 6.14 | Impact Factor (2014): 5.611}

synthesis. The lost patches are synthesized along the user specified curves. For single curve simple dynamic programming is useful. Method is good for less no of objects, as no of objects increases optimization becomes difficult. The semi automatic techniques [10] are used to repair films whereas this technique results in blur effect in image. From above discussion it is clear that for large area more time required. To overcome this, Oliviera et al. [24] proposed a fast digital inpainting based on an isotropic diffusion model.

\subsection{Wavelet transform}

The algorithm [8] presented the technique with the help of the wavelet transform. The evaluation of the best universal structure of damaged regions in addition to shape and texture properties. To deal with the fact of multi resolution analysis, data partition along with the statistical properties the wavelet transform has better image demonstration quality. Wavelet transform try to maintain the human perception. The image inpainting process is applied in the wavelet domain by in view of both scaling and wavelet coefficient in the target region. The algorithm of wavelet transform is capable of improving texture structure and image quality. Nevertheless, difficulties in this algorithm mask for regions are calculated manually.

\section{Discussion}

In this section, the experimental results confirm combined data hiding and image compression scheme is effective and feasible. The joint data hiding and image compression scheme based on SMVQ [7] shows its good ability to hide the secret data in low bit rate. Also it is having very high hiding capacity, low distortion and high visual quality. Whereas, VQ [17] is most popular data hiding scheme cause of their simplicity and cost effective implementation. Encoding and decoding is faster for VQ as compared to JPEG/JPEG2000 where as VQ is limited for visual quality and compression ratio [1]. Data hiding techniques are used for authentication, information security, fine art work, law enforcement, military use, medical applications etc.

One of the main problems with inpainting technique is the reproduction of large textured regions, take more time. Most of the algorithms employ well for tiny scratch region. Furthermore, the modified inpainting algorithms are the improved visual quality and applied for large regions. Application of digital inpainting includes the restoration of smashed photographs, elimination or insertion of objects, red eye removal etc.

\section{Conclusion}

This paper represents a review of combined data hiding techniques and compression algorithms and types of digital inpainting. Side match vector quantization is modified technique of vector quantization. Reversible data hiding techniques are ideal for military and medical imaging application where the loss of quality is always unbearable. LSB technique is simple way to embed the secret data into the image and also it can be easily decoded at receiver end.
Though, it is time consuming process but it gives better security.

This paper represents review of various digital Inpainting techniques. The performance of different techniques is compared, in terms of time required for inpainting and size of the region. The components of image inpainting maintain the visual quality. An efficient algorithm can decrease time requirement and computational cost.

\section{References}

[1] C.-C. Chang and W.-C. Wu, "A steganographic method for hiding secret data using side match vector quantization," IEICE Trans. Inf. Syst., vol. E88-D, no. 9, pp. 2159-2167, 2005.

[2] M. Jo and H. D. Kim, "A digital image watermarking scheme based on vector quantization," IEICE Trans. Inf. Syst., vol. E85-D, no. 6, pp. 10541056, Jun. 2002.

[3] P.-Y. Tsai, M.-H. Lin, and C.-C. Chang, "An adaptive steganographic scheme for color image," Fund. Inf., vol. 62, no. 3-4, pp. 275-289, Aug. 2004.

[4] J. M. Barton, "Method and apparatus for embedding authentication information within digital data", U.S. Patent 5646 997, Jul. 8, 1997.

[5] J. Fridrich, M. Goljan, and R. Du, "Invertible authentication watermark for JPEG images," in Proc. ITCC , Las Vegas, NV, Apr. 2001, pp. 223-227.

[6] T. Kim, "Side match and overlap match vector quantizers for images," IEEE Trans. Image Process., vol. 1, no. 4, pp. 170-185, Apr. 1992.

[7] Chin-Chen Chang, Fellow, IEEE, Wei-Liang Tai, and Chia-Chen Lin," A Reversible Data Hiding Scheme Based on Side Match Vector Quantization ", IEEE transactions on circuits and systems for video technology, vol. 16, no. 10, october 2006.

[8] Dong wookcho and Tien D. Bui "Image In painting Using Wavelet-Based Inter and Intra-Scale Depedency” IEEE Transactions on Image Processing, 2008.

[9] H. W. Tseng and C. C. Chang, "High capacity data hiding in JPEG compressed mages," Informatica, vol. 15, no. 1, pp. 127-142, 2004

[10]A. Bugeau, M. Bertalmío, V. Caselles, and G. Sapiro, "A comprehensive framework for image inpainting," IEEE Trans. Image Process., vol. 19, no. 10, pp. 26342644, Oct. 2010.

[11] M. Bertalmio, G. Sapiro, V. Caselles and C. Ballester, "Image inpainting," Computer Graphic (SIGGRAPH 2000), pp. 417-424, July 2000.

[12] Criminisi, A., P'erez, P., Toyama, K.: Region filling and object removal by examplar based image inpainting. IEEE Trans. On Image Processing 13 (2004) 1200-1212.

[13]Rajul Suthar, et. Al, Int. Journal of Engineering Research and Applications www.ijera.com, ISSN: 2248-9622, Vol. 4, February 2014, pp.85-88

[14] Khushbu A. Panchal , Dr.Mehfuza Holia," A Survey: Different Techniques of Video Inpainting”, International Journal of Innovative Research in Computer and Communication Engineering, Vol. 2, Issue 2, February 2014.

[15] Wei-Qi Yan; Kankanhalli, M.S., "Erasing video logos based on image inpainting," Multimedia and Expo, 
2002. ICME '02.Proceedings. 2002 IEEE International Conference on , vol.2, no., pp.521,524 vol.2, 2002.

[16] G. Xuan, J. Zhu, J. Chen, Y.-Q. Shi, Z. Ni, and W. Su, "Distortionless data hiding based on integer wavelet transform," Electron. Lett., vol. 38, no. 25, pp. 16461648, Dec. 2002.

[17]B. Yang, Z.-M. Lu, and S.-H. Sun, "Reversible watermarking in the VQ-compressed domain," in Proc. 5th VIIP, Benidorm, Spain, Sep. 2005, pp. 298-303.

[18] W. C. Du and W. J. Hsu, "Adaptive data hiding based on VQ compressed images," IEE Proc. Vis., Image Signal Process., vol. 150, no. 4, pp. 233-238, Aug. 2003.

[19]C. C. Chang and W. C. Wu, "Hiding secret data adaptively in vector quantisation index tables," IEE Proc. Vis., Image Signal Process., vol. 153, no. 5, pp. 589-597, Oct. 2006.

[20] C. C. Lin, S. C. Chen, and N. L. Hsueh, "Adaptive embedding techniques for VQ-compressed images," Inf. Sci., vol. 179, no. 3, pp. 140-149, 2009.

[21] C. H. Hsieh and J. C. Tsai, "Lossless compression of VQ index with search-order coding," IEEE Trans. Image Process., vol. 5, no. 11, pp. 1579-1582, Nov. 1996.

[22] A.C. Kokaram, R.D. Morris, W.J. Fitzgerald, P.J.W. Rayner. Detection of missing data in image sequences. IEEE Transactions on Image Processing 11(4), 14961508, 1995.

[23] A. Hirani and T. Totsuka. Combining Frequency and spatial domain information for fast interactive image noise removal. Computer Graphics, pp. 269-276, SIGGRAPH 96, 1996.

[24] M. Oliviera, B. Bowen, R. Mckenna, and Y.-S. Chang. Fast Digital Image Inpainting. In Proc. Of Intl. Conf. On Visualization, Imaging And Image Processing (VIIP), Page 261266, 2001.

[25]C. Braverman. Photoshop retouching handbook. IDG Books Worldwide, 1998.

\section{Author Profile}

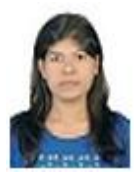

Sarita S. Kamble received the B.E. degree in Electronics and Tele-communication from the Savitribai Phule Pune University, Pune Maharashtra, India, in 2013. Currently, she is a pursuing Master of Engineering from Imperial college of Engineering and Research, Wagholi, Pune, Maharashtra SPPU University. Her current research interests include, "Joint Data hiding and compression scheme based on Side match Vector Quantization and Digital Inpainting." 